\title{
Brain Tumor Segmentation and Classification using KNN Algorithm
}

\author{
Suhartono, Phong Thanh Nguyen, K. Shankar, Wahidah Hashim, Andino Maseleno
}

\begin{abstract}
Image processing plays a vital role in MRI image processing. MRI images are widely used in medical fields for analysis and detection of tumour growth from the body. There are varieties of efficient brain tumour detection and segmentation methods have been suggested by various researchers for efficient tumour detection. Existing methods encounter with several challenges such as detection time, accuracy and quality of tumour. In this review paper, we are presenting a study of various tumour detection methods for MRI images. A comparative analysis has been also performed for various methods.SAR images are the high resolution images which cannot be collected manually. In this work, we identified the SAR images randomly from web with different region inclusions. The regions in an image include water area, land area and the mountain area. The implementation of proposed model is done in MATLAB environment.
\end{abstract}

Keywords: Cancer Detection, kNN Algorithm, Segmentation.

\section{I.INTRODUCTION}

Digital Image processing assumes an imperative job in MRI picture handling. X-ray pictures are generally utilized in restorative fields for examination and identification of tumor development from the body. There are assortments of productive cerebrum tumor identification and division techniques have been proposed by different analysts for effective tumor discovery. Existing strategies experience with a few difficulties, for example, location time, precision and nature of tumor. In this survey paper, we are introducing an investigation of different tumor identification techniques for MRI pictures. A similar examination has been likewise performed for different strategies. SAR pictures are the high goals pictures which can't be gathered physically. In this work, we distinguished the SAR pictures arbitrarily from web with various locale considerations. The districts in a picture incorporate water region, land territory and the mountain region.

Revised Manuscript Received on July 22, 2019.

Suhartono, Informatics andComputer Education Study Program, Universitas Negeri Makassar, Indonesia.

Phong Thanh Nguyen, Department of Project Management, Ho Chi Minh City Open University, Vietnam.

K. Shankar, Department of Computer Applications Alagappa University, India.

Wahidah Hashim, Institute of Informatics and Computing Energy, Universiti Tenaga Nasional, Malaysia.

Andino Maseleno, Institute of Informatics and Computing Energy, Universiti Tenaga Nasional, Malaysia.

\section{Binary image \\ II. TYPES OF DIGITAL IMAGES}

The binary images are one bit images. In binary image the value of each pixel is either black or white. The binary image is a combination of two possible values either 0 or 1 . In such type of images only one bit is used to represent each pixel. In this type of images black color is represented by ${ }^{\prime} 0$ ' and white color by ' 1 '.

Grayscale image

Gray scale image is 8 bit image. In grayscale image each pixel is represented by a gray level. There are 256 gray levels. The gray level range is from 0 to 255 where ' 0 ' gray level represents black color and ' 255 'represents white color. Each pixel is a combination of eight bits in gray scale image.

\section{True color or RGB image}

RGB image is a 24 bit image where every pixel has a particular color. This color in the image is described by the combination of three matrices that represents red, green and blue component values. The combination of these three components gives $16,777,216$ different colors when each of the color has a gray scale range from 0 to 255 .Every pixel in true color image is a combination of three gray scale values. Figure 1 shows color model of primary and secondary colors.

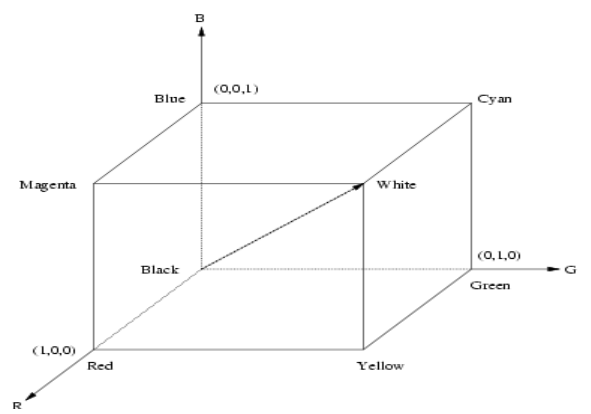

Fig.1: Color model

The RGB color model shows Red, Green and Blue colors are primary colors. When Red, Green and Blue colors set to $(0,0,0)$, it gives a black color and when they set to $(1,1$, 1),it gives a white color. The secondary colors like Magenta, Cyan, Yellow, Black and White are obtained by the combination of primary colors.

Figure 2 shows the secondary colors obtained from primary colors. Yellow, white, magenta etc are the secondary colors.

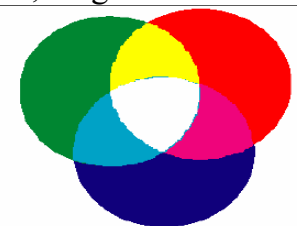

Fig.2: Primary and secondary color

Figure 3 shows different types of image formats 


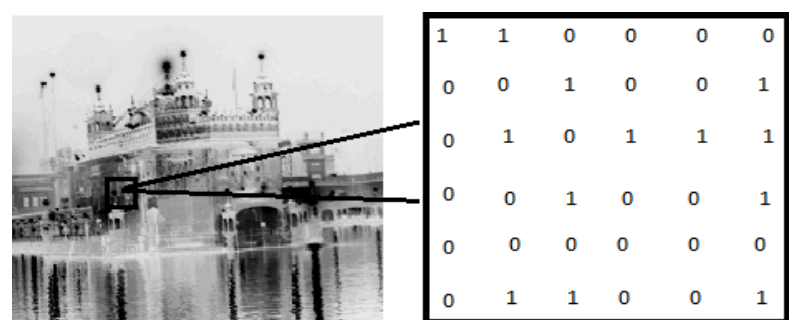

(a)

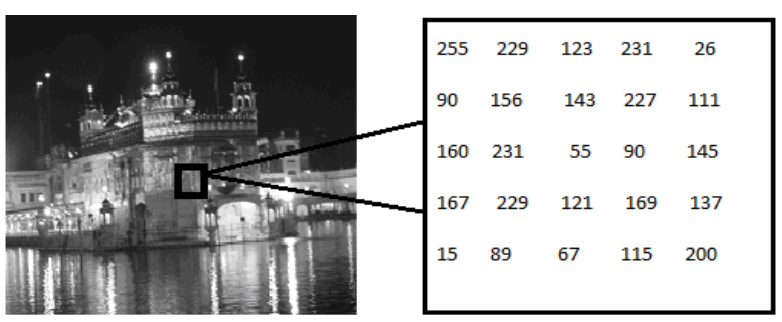

(b)

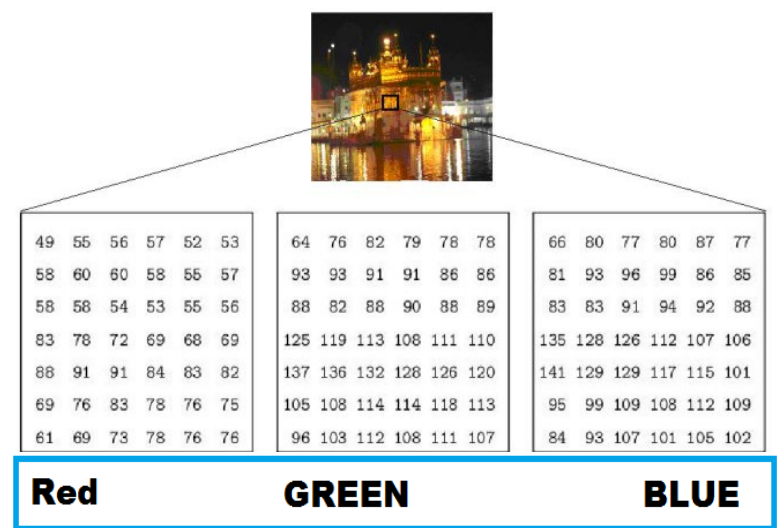

Fig.3: Types of Image (a) binary image (b) grayscale image (c) color image

Figure 'a' shows a binary image (black and white image) represented by the combination of 0 and 1 value of pixels. Figure ' $b$ ' shows a gray scale image having different gray level values varies from $0-255$ and the image shown in figure ' $c$ ' is a color image represented by the combination of red, green and blue gray levels.

\section{IMAGE SEGMENTATION}

\section{Overview}

The Segmentation is process that separate object an image. By using segmentation process it is possible to identity a particular object from an image. Segmentation is a technique that separate out foreground and background of an image. Segmentation algorithm is generally based on basic two properties of gray levels that are discontinuity and similarity. The discontinuity is based on difference in intensity values of pixels in an image. The discontinuities between gray levels regions are used to detect contours within an image. The similarity between the intensity values of pixels also useful for identification of objects based on some predefined criteria's applicable to both static and dynamic images. In segmentation input images are inputs and outputs are attributes extracted from those images. The segmentation process stops when object of interest in an image is isolated. The different segmentation techniques based on discontinuities are point segmentation, line segmentation and edge segmentation.

\section{Design Steps}

(1) Set the initial threshold $\mathrm{T}=$ (the maximum value of the image brightness + the minimum value of the image brightness) $/ 2$.

(2) Using $\mathrm{T}$ segment the image to get two sets of pixels B (all the pixel values are less than $\mathrm{T}$ ) and $\mathrm{N}$ (all the pixel values are greater than $\mathrm{T}$ );

(3) Calculate the average value of $\mathrm{B}$ and $\mathrm{N}$ separately, mean $\mathrm{ub}$ and un.

(4) Calculate the new threshold: $\mathrm{T}=(\mathrm{ub}+\mathrm{un}) / 2$

(5) Repeat Second steps to fourth steps up to iterative conditions are met and get necessary region from the brain image.

\section{Proposed Method}

MRI Brain image Classification and anatomical structure analysis are proposed based on,

(1) PNN-RBF Network for classification

(2) Fuzzy clustering for tumor detection and structural analysis

\section{SYSTEM ARCHITECTURE}

The method include brain tumor segmentation using optimized k-means and genetic algorithm.

For clustering the Optimized k-means algo is used. The classification and feature extraction is handeled by Genetic algorithm.At last the tumor is extracted from the MRI image. After extraction its exact place and the size can be determined in grayscale. 


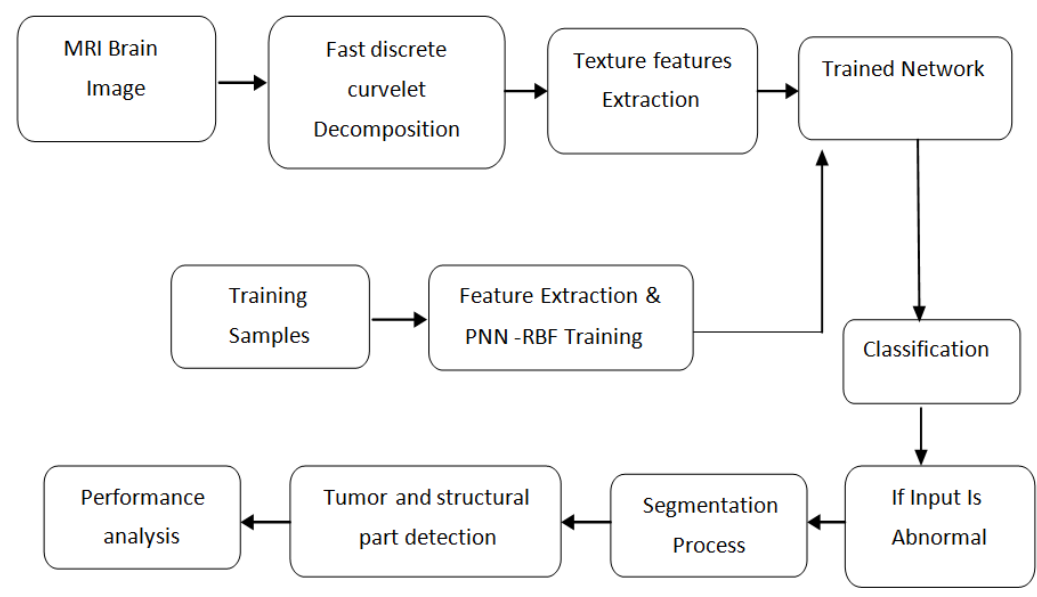

Fig.4: Block Diagram of Proposed System

\section{Developing GUI}

The GUI in Matlab can be developed with the help of a number of tools and functions available in the Matlab library. There are many GUI tools for frontend.

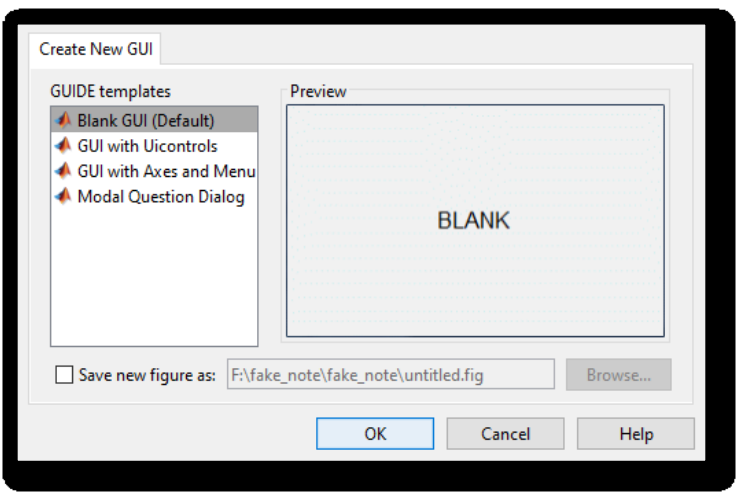

Fig. 5: GUIDE Quick Start Dialog

The figure 5 shows the basic guide using start dialog. Now the further steps can be followed according to the instructions given in the dialog box.

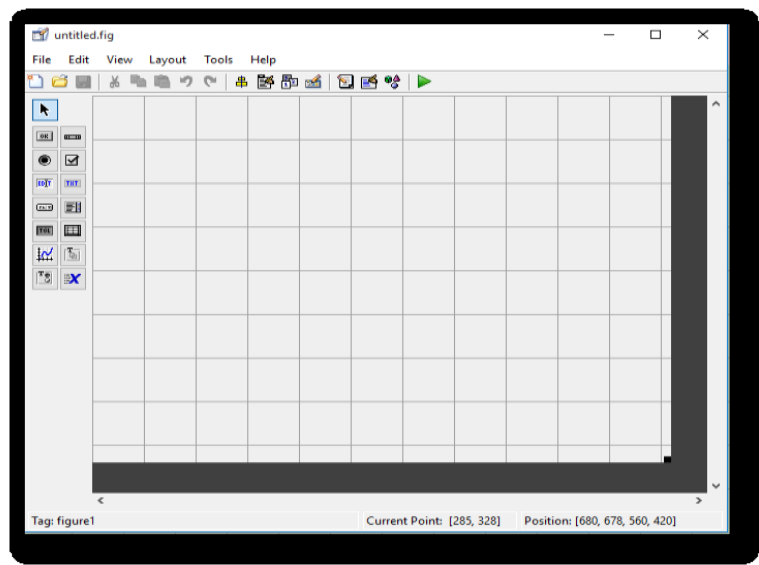

Fig. 6: The Layout Edit

There are two types of file where first file is matlab code file with extension .m and another file is layout file which contains extension .fig.

\section{SYSTEM EXECUTION DETAILS}

It is explain the step by step executions of the given system are as follows

\section{Output of Proposed System}

This section provides the outcome of the proposed system. Step by step outcome is presented below in S.

\section{Input Image with its curvelet Decomposition}

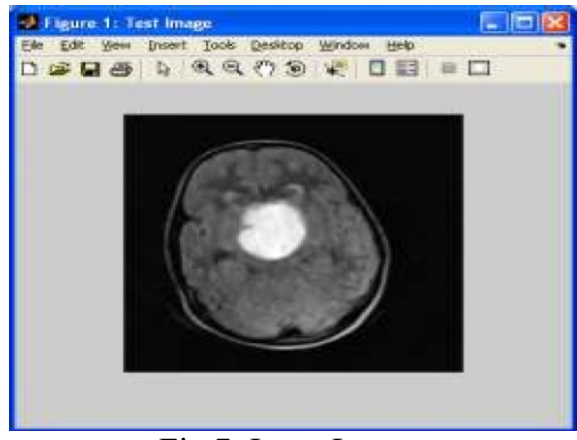

Fig 7: Input Image

Here the input MRI image is taken for the implementation as shown in figure 7.

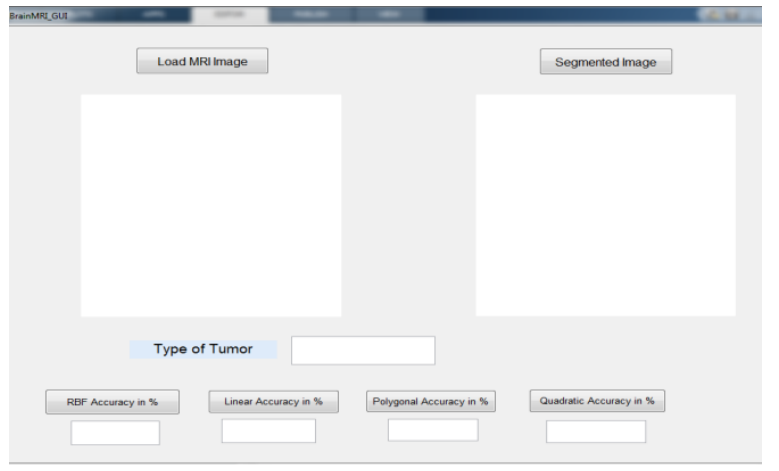

Fig 8: GUI of the System

In figure 8 there are two windows. One shows input image and second shows the output image.

Published By:

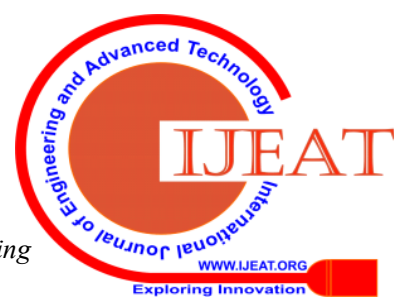




\section{IMAGE SEGMENTATION}

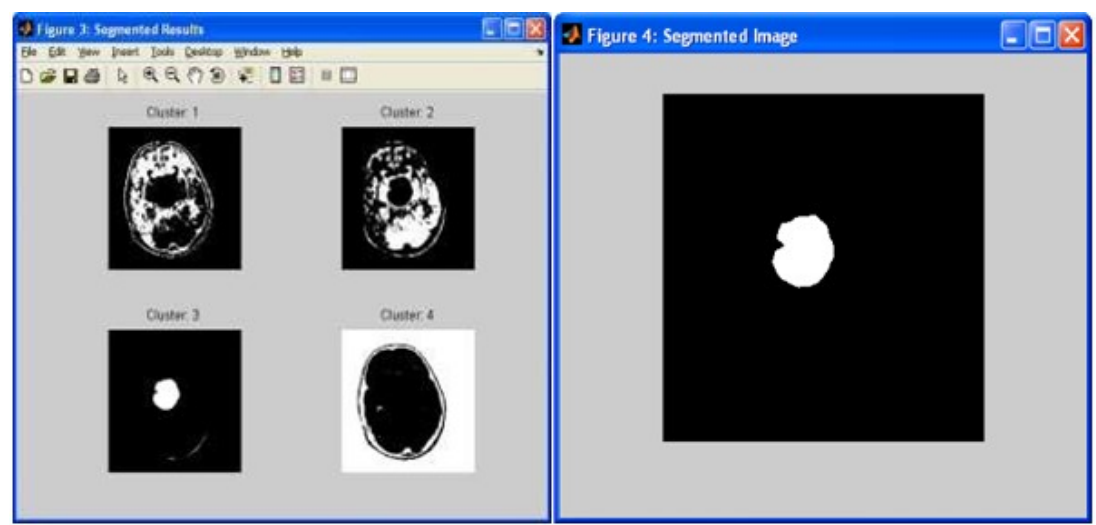

Fig 9 : MRI Brain with Benign Case

The Figure 9 shows the segmentation of the input image. The Bengin Tumor plot is shown in the output window.

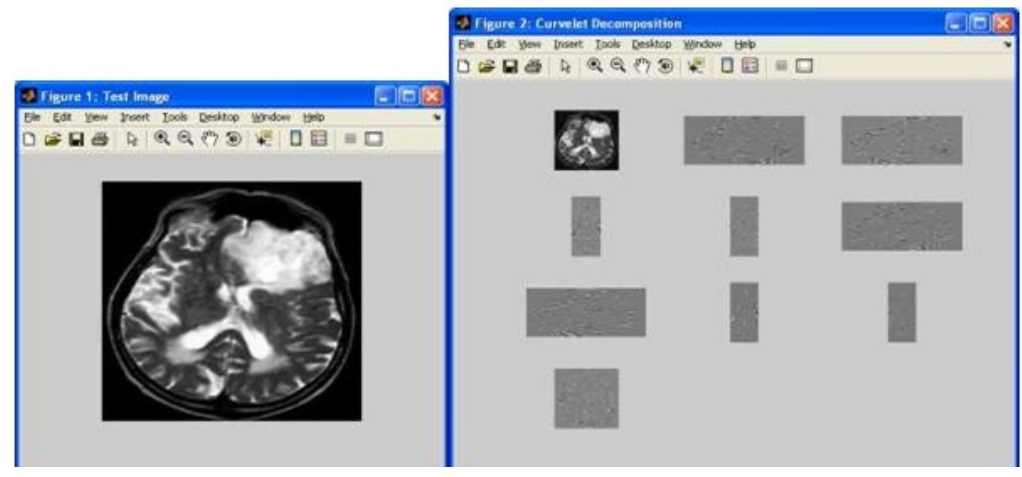

Fig. 10: MRI Brain with Malignant Case

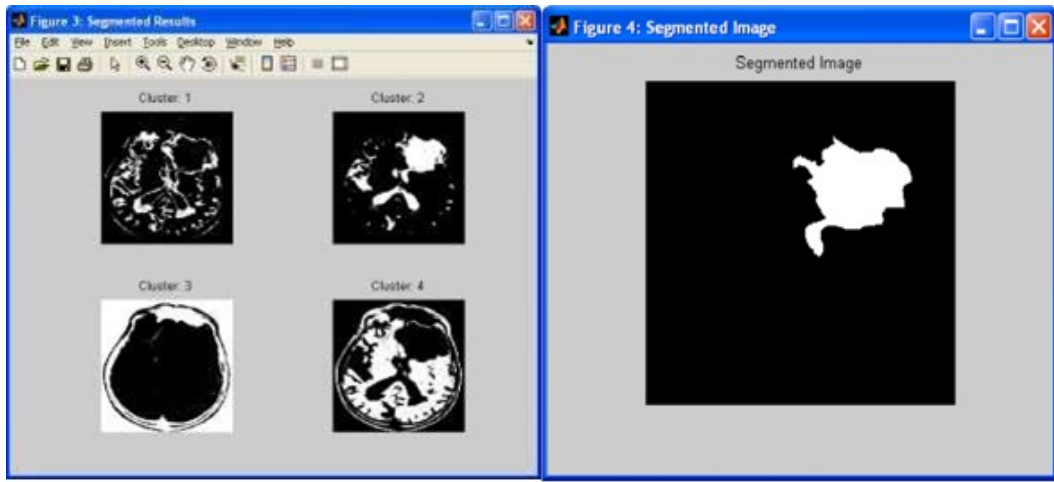

Fig.11: Malignant Tumor Image

The Figure 11 shows the segmentation of the input image.

The Bengin Malignant plot is shown in the output window. 


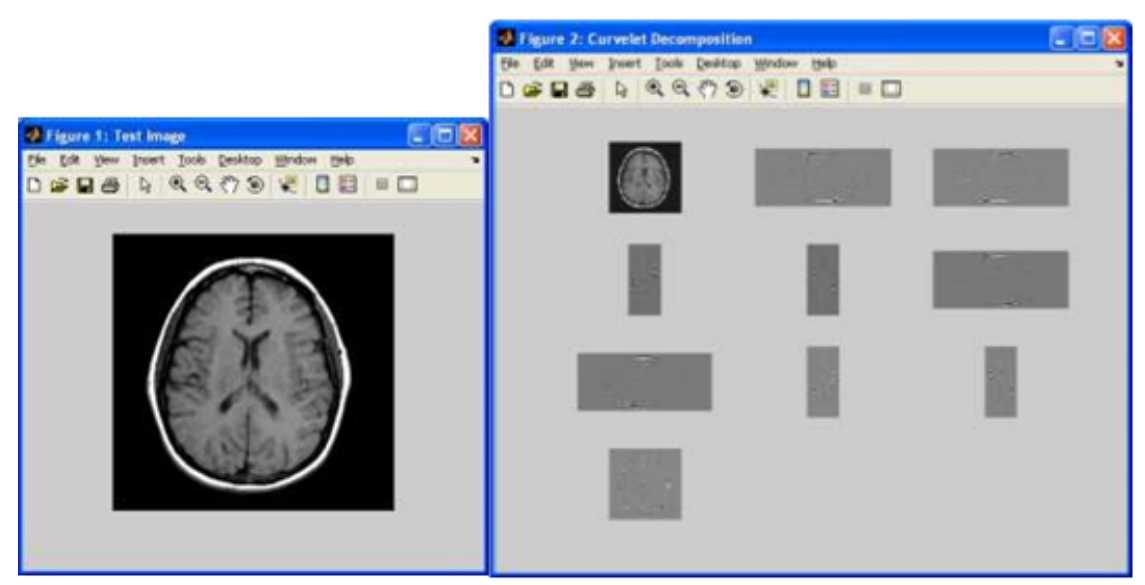

Fig. 12: MRI Brain with Normal Case

The figure 12 shows the normal image of brain. So in the

IMAGE SEGMENTATION output window there is no plots or tumor segmentation.

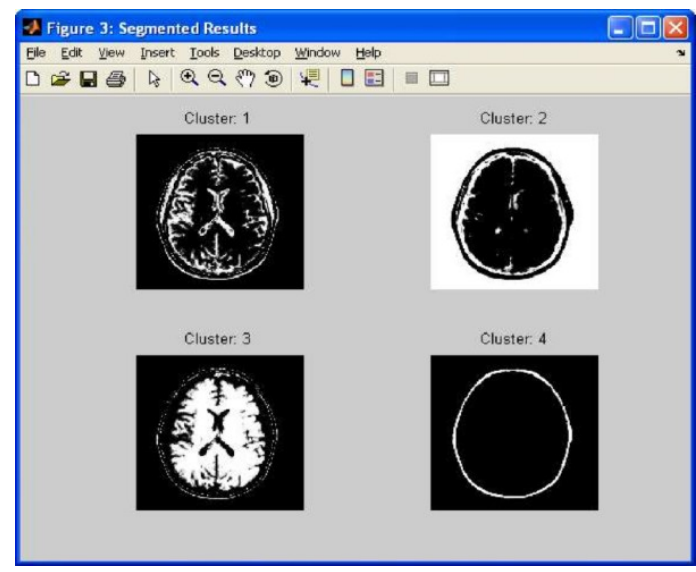

Fig. 13: Segmented Image

The input image segmentation process is shown in the figure 13. The segmentation is shown cluster wise.

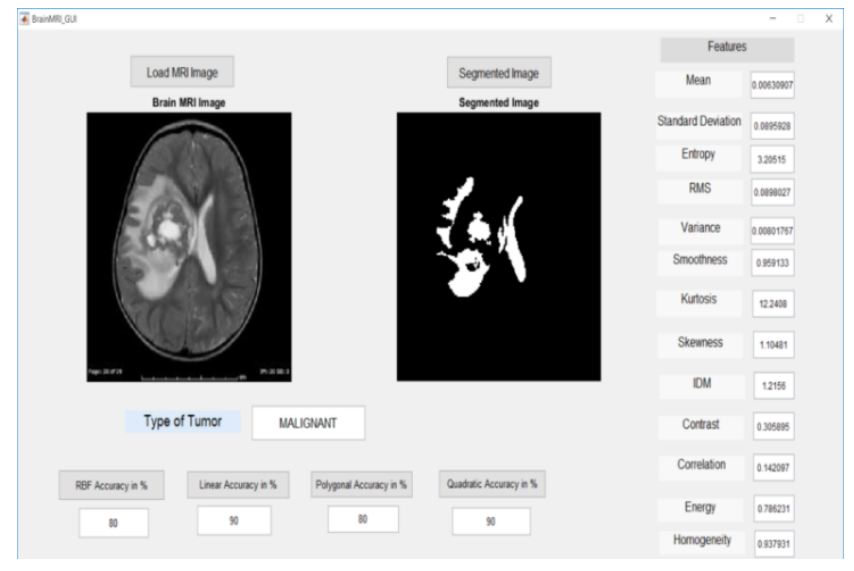

Fig. 14: Final Output of the System

\section{PERFORMANCE MEASUREMENT}

The performance of the given system measured by using the following table that given various condition for outputs. They give the multiple images of brain MRI that are detected by the system. The first condition if one brain MRI image is provided from the dataset and system detected it as tumor is present then it is considered as the true positive.
Precision $=$ Relevant instance $/$ Retrieve instance

Recall $=$ Relevant instance that have been retrieve $/$ Total amount of instance

Published By:

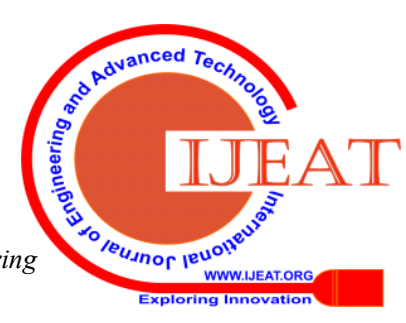




\section{EVALUATION RESULT}

The figure 15 shows the result of precision and recall of the brain MRI image of the current system as well as existing system according to the accuracy from the 23 images which are taken from the dataset.

The graph based on Linear accuracy in figure 15 shows the comparison of accuracy, precision and recall between existing work and proposed work.

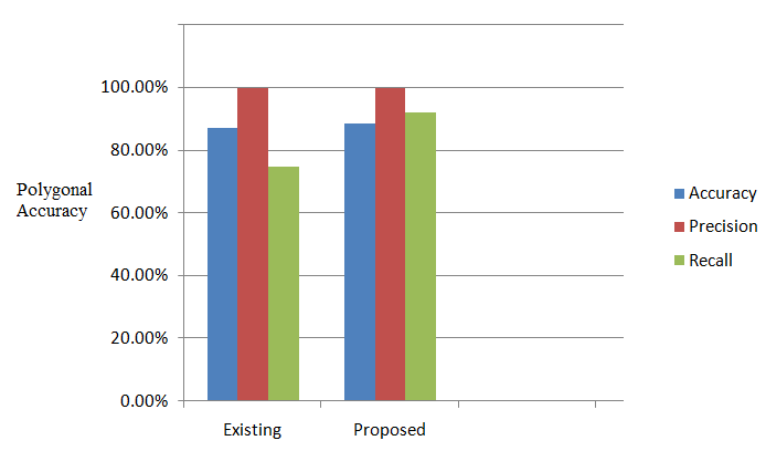

Fig.15: Graph based on Polygonal Accuracy

The graph based on Quadractic accuracy in figure 15 shows the comparison of accuracy, precision and recall between existing work and proposed work.

\section{CONCLUSION}

Existing methods encounter with several challenges such as detection time, accuracy and quality of tumour. In this review paper, we are presenting a study of various tumour detection methods for MRI images. A comparative analysis has been also performed for various methods. SAR images are the high resolution images which cannot be collected manually. In this work, we identified the SAR images randomly from web with different region inclusions. The regions in an image include water area, land area and the mountain area. The implementation of proposed model is done in Matlab environment.Currently our proposed system works on only two types of brain tumor. In future, the proposed system can be improved by adding different type tumor for classification. Also the accuracy of the proposed system can be improved by including different dataset available from doctors throughout the world. The proposed system can also be further extended for detecting cancer and other tumors.

\section{REFERENCES}

1. B. H. Menze, K. Van Leemput, D. Lashkari, M. A.Weber, N. Ayache, and P. Golland, "A generative model for brain tumor segmentation in multimodal images," in Proc. Int. Conf. Med. Image Comput. Comput.-Assisted Intervent., 2010, pp. 151159.

2. C. Cortes and V. N. Vapnik, "Support vector networks," Mach. Learn., vol. 20, no. 3, pp. 273297, 2010.

3. W. E. Phillips, R. P. Velthuizen, S. Phuphanich, L. O. Hall, L. P. Clarke, and M. L. Silbiger, "Application of fuzzy c-means segmentation technique for tissue differentiation in MR images of a hemorrhagic glioblastoma multiforme," Magn. Reson. Imag., vol. 13, no. 2, pp. $277290,2010$.

4. A. Mehnert and P. Jackway, "An improved seeded region growing algorithm," Pattern Recognit. Lett., vol. 18, no. 10, pp. $10651071,2010$.

5. R. Adams and L. Bischof, "'Seeded region growing," IEEE Trans. Pattern Anal. Mach. Intell., vol. 16, no. 6, pp. 641647, Jun. 2010.
6. R. Beare, "Regularized seeded region growing," in Proc. 6th Int. Symp. Math. Morphol., 2002, pp. 9199.

7. A. Yezzi, S. Kichenassamy, A. Kumar, P. Olver, and A. Tannenbaum, "A geometric snake model for segmentation of medical imagery," IEEE Trans. Med. Imag., vol. 16, no. 2, pp. 199209, Apr. 1997.

8. C. Li, C. Xu, C. Gui, and M. D. Fox, 'Distance regularized level set evolution and its application to image segmentation," IEEE Trans. Image Process., vol. 19, no. 12, pp. 32433254, Dec. 2010.

9. B. N. Li, C. K. Chui, S. H. Ong, and S. Chang, "'Integrating FCM and level sets for liver tumor segmentation," in Proc. 13th Int. Conf. Biomed. Eng., Berlin Germany, 2009.

10. O. Fajarianto, M. I. Setiawan, A. Mursidi, D. Sundiman, and D. A. P. Sari, "The Development of Learning Materials for Introduction of Animals in Early Childhood Using Augmented Reality," 2018, pp. 722-727.

11. D. A. Puspito Sari, I. Listiyowati, T. Nefianto, and Lasmono, "The Discrepancy between The Programs and Disaster Management Policy in Klapanunggal District, Bogor, West Java," IOP Conf. Ser. Earth Environ. Sci., vol. 135, no. 1, p. 012011, Mar. 2018.

12. Lydia, E.L., Kumar, P.K., Shankar, K., Lakshmanaprabu, S.K., Vidhyavathi, R.M. and Maseleno, A., 2018. Charismatic Document Clustering Through Novel K-Means Non-negative Matrix Factorization (KNMF) Algorithm Using Key Phrase Extraction. International Journal of Parallel Programming, pp.119.

13. Abadi, S., Teh, K.S.M., Nasir, B.M., Huda, M., Ivanova, N.L., Sari, T.I., Maseleno, A., Satria, F., Muslihudin, M. Application Model of K-Means Clustering Insights into Promotion Strategy of Vocational High School. International Journal of Engineering \& Technology, 7(2.27), pp.182-187.

14. Lydia, E.L., Kumar, P.K., Shankar, K., Lakshmanaprabu, S.K., Vidhyavathi, R.M. and Maseleno, A., 2018. Charismatic Document Clustering Through Novel K-Means Non-negative Matrix Factorization (KNMF) Algorithm Using Key Phrase Extraction. International Journal of Parallel Programming, pp.119. 